\title{
Sea glider guidance around a circle using distance measurements to a drifting acoustic source
}

\author{
Jan Sliwka ${ }^{1,2}$, Benoît Clement ${ }^{1,2}$ and Irvin Probst ${ }^{1,2}$
}

\begin{abstract}
This paper describes a simple yet robust sea glider guidance method in a constellation of Lagrangian drifters under the polar ice cap. The glider has to perform oceanographic measurements, mainly conductivity, temperature and depth, in the area enclosed by the drifters and can not rely on GNSS (Global Navigation Satellite System) positionning data as the polar ice cap makes it impossible to surface. The originality of the presented method resides in 2 points. First, a very simple PID (Proportional, Integral and Derivative) controller based on a basic kinematic model is tuned. Second, the method does not use a localization algorithm to estimate state space model data but interval analysis methods are performed to bound the errors in range to the transponder and its derivative. Moreover, only one acoustic beacon is used. Validation is then performed through simulations.
\end{abstract}

\section{INTRODUCTION}

Deploying a constellation of drifting floating systems performing oceanographic measurements under the polar ice cap is part of the ACOBAR (ACoustic technology for OBserving the ARctic) project (see [1]) as seen in Fig. I.

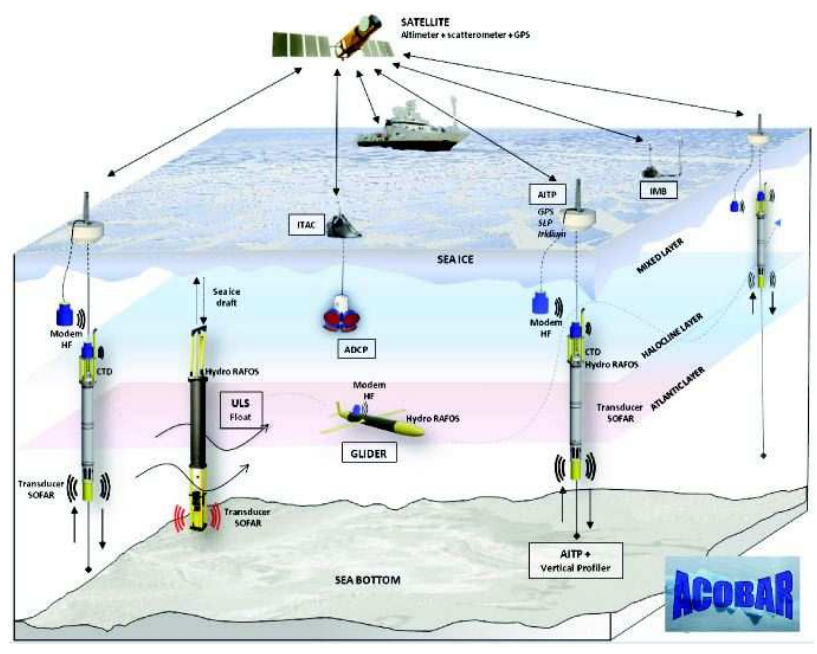

Fig. 1. ACOBAR project overview

In order to fill the gap in data between the Lagrangian drifters (denoted as "floats" from now on), a sea glider has been chosen to navigate in between the floats. The floats are moored into the ice cape and used as acoustic beacons using RAFOS emitters (SOund Fixing And Ranging, SOFAR spelled backwards) ([2]) which sends a chirped acoustic

\footnotetext{
${ }^{1}$ ENSTA Bretagne / Ocean Sensing and Mapping Team, 2 rue F. Verny, 29200 Brest, France firstname.name at ensta-bretagne.fr

2 Lab-STICC UMR CNRS 6285
}

signal at well known time slots. The frequency of this signal is usually in the $0-2 \mathrm{kHz}$ band, as the expected width of the float network will be below $100 \mathrm{~km}$, and as lower frequency emitters are very heavy and power consuming, the 1560 $\mathrm{Hz}$ RAFOS emitters are used here. Knowing the time of emission of several emitters, and measuring the time of arrival on a device one wishes to localize, is enough to get ranging measurements to each emitter and therefore localize the device in the emitters frame. On the other hand if one knows the position of several receiving devices it is possible to localize the emitters. Such low frequency signals can be detected at ranges up to $100 \mathrm{~km}$ for $1560 \mathrm{~Hz}$, or even more for lower frequencies, but they can barely transmit any data.

The glider is supposed to navigate between the floats in order to collect oceanographic data, and once it gets close enough to a float (at most $10 \mathrm{~km}$ ) a high frequency acoustic modem is used to download the data from the floats. Then the floats use their Iridium modems to relay the data.

The RAFOS localization has been proven to be efficient for decades but it puts a lot of constraints on the emitters network. There are two major issues:

1) The float positions are to be known by the glider in order to compute its position, but the floats are drifting as the ice cap moves. As the glider can not surface to use satellite data networks the only way to transmit the floats positions is to use an acoustic link.

2) Usual localization methods, such as trilateration [3], require the glider to have at least three beacons in range, but once again the float are moving and it is not possible to guarantee that the ice movements will not tear the float network apart.

One could think of using unary data coding between successive RAFOS emissions ${ }^{1}$ to transmit data to the glider, this might solve the first issue but it will not prevent the network from getting too wide. Furthermore more issues are making real-time autonomous positioning an even more daunting task, for instance clock drifts, local variations in the speed of sound, and so on.

Finally it is not possible to rely on a compass close to the magnetic pole, therefore even if the positionning problems (i.e. navigation) could be solved the guidance would not be an easy task. In order to solve these problems, this paper proposes a simple and robust guidance algorithm using the ranging data described above. Our approach is to split the localization of the glider, which is mandatory to geolocalize

\footnotetext{
${ }^{1}$ For instance a 10 seconds delay between two successive RAFOS signals means a predefined variable has now the value "10".
} 
the oceanographic measurements, and the guidance task which objective is to follow a given trajectory. The idea is to navigate around only one float, called the master float, and store the other floats' ranging data for post-processing once the data are transmitted to the operators. Doing this removes the need to know the glider position in real-time and gives the operators much more time and computing power to perform fine adjustments on the positionning data, therefore increasing the scientific value of the oceanographic measurements. Finally less tasks performed by the glider onboard software means a lower power consumption therefore longer missions.

The simplest motion possible around the master float is to follow a circular trajectory of a setpoint radius $r$. This radius can be changed during the mission in order to cover a wider area using unary coding as explained above. Since the trajectory is circular, the problem is invariant with rotation so the task can be performed using a gyroscope instead of a compass which is a critical point for polar navigation.

\section{GUIDANCE USING A SINGLE BEACON}

Several authors considered navigation and guidance of AUV (Autonomous Underwater Vehicle) using ranging data to a single beacon (see for example [4], [5],[6], [7] or [8] and references inside). This kind of guidance consists in the AUV (or beacon) localization in order to navigate around the beacon. The main issue is that it is not possible to localize the beacon with only one measurement. The AUV has to make ranging measurements from different places while moving in order to perform the navigation. As a consequence the displacements of the AUV has to be known with maximal accuracy (using dead reckoning for example) in order to create a solid baseline for the trilateration. The AUV has to perform appropriate maneuvers to make the localization efficient. In [5], it is claimed that the trajectory which maximizes the information given by the measured ranges is when the vehicle describes a circle centered at the beacon.

In this article we propose a different approach which consists on using a simple regulator based on intuitive behavior. The regulator should be simple i.e. it includes no observation of the different unknown states of the problem. The method can be then validated using the viability theory [9] or using Monte Carlo testing methods (take several random initial conditions and make statistics on convergence).

The guidance algorithms are supposed to be:

- user friendly (few configuration parameters to make tuning easy);

- robust to:

- variable and unknown perturbations (here sea current velocity and master float drift velocity),

- measurement noise,

- missing or irregular measurements,

- outliers.

\section{MOTION EQUATIONS}

A sea glider is an AUV which moves without any propeller (see [10] or [11] for examples). ENSTA Bretagne has developed its own glider [12] and [13] presented in Fig. III.
Vehicle pitch and roll are controlled by two mobile masses and the buoyancy regulation is performed by a ballast (a pump or a buoyancy engine). The glider cycle consists of:

- making it sink while pitching down, until it reaches the desired depth;

- making it surface while pitchning upwards.

The yaw rate $\omega$ is proportional to the roll $\phi$.

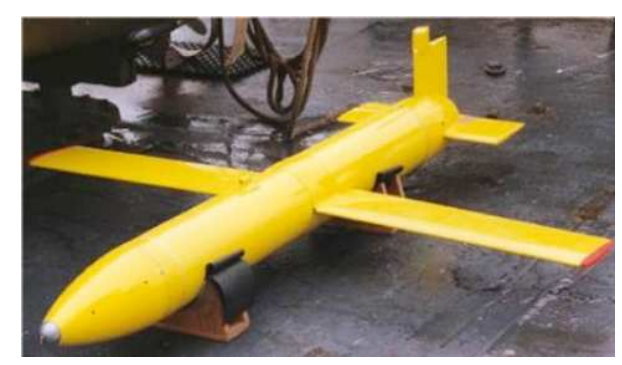

Fig. 2. ENSTA Bretagne Glider: Sterne

In this paper, only the $2 \mathrm{D}$ problem is considered. Indeed, even if the glider depth is time varying, as it is perfectly known, the ranging measurements are depth compensated and then we only consider the cylinder projection (i.e. the $2 \mathrm{D}$ circle). Moreover, the speed of the vehicle $v_{g}$ is considered as a constant through time by neglecting the transitions during the gliding cycle. Fig. 3 shows the different parameters which are used in the article.

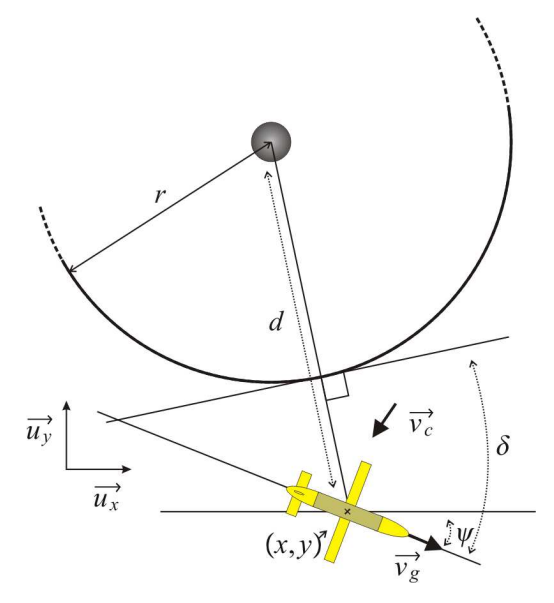

Fig. 3. The different parameters of the problem

\section{A. Notations}

Denote by:

- $d$ the distance between the glider and the float;

- $r$ the radius of the circle;

- $\Delta r$ the width of the regulation zone;

- $\psi$ the heading of the glider;

- $v_{g}$ the velocity of the glider relative to water;

- $\overrightarrow{v_{c}}$ the velocity vector of the relative current between the beacon and the glider $\left(v_{c}=\left\|\overrightarrow{v_{c}}\right\|\right.$ and $\left.\alpha=\arg \left(\overrightarrow{v_{c}}\right)\right)$;

- $\delta$ the angle between glider's heading and the tangent to the circle perpendicular to the line glider-Beacon;

- $\omega$ general angular velocity of the glider. 


\section{B. Simulation oriented motion equations}

For the simulation, we chose very simple equations to model the motion of the glider in the Cartesian workspace.

$$
\begin{aligned}
& \dot{x}=v_{g} \cos \psi+v_{c} \cos \alpha \\
& \dot{y}=v_{g} \sin \psi+v_{c} \sin \alpha \\
& \dot{\psi}=\omega .
\end{aligned}
$$

Note that these equations can be applied to other types of robots thus our guidance approach can be easily transposed.

\section{Regulation oriented motion equations}

Let us first consider the line following problem as shown on Fig. 4. The dashed line denotes the trajectory the glider has to follow, the bold line is the reference for the range measurements. Actually this problem is the same as the circle following problem when the radius $r$ tends to infinity (or is big enough) as we will show it later.

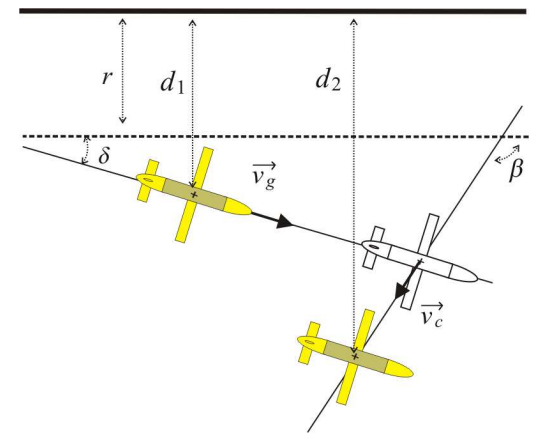

Fig. 4. Line following

Between the two consecutive glider positions denoted on Fig. 4, one can easily deduce the following state space model:

$$
\begin{aligned}
& \dot{d}=v_{c} \sin \beta+v_{g} \sin \delta \\
& \dot{\delta}=\omega .
\end{aligned}
$$

with $\beta$ being the angle between the current and the line to be followed. Furthermore the line following problem implies that $\delta=\psi+$ Constant which obviously gives $\dot{\psi}=\omega$. This last equation is therefore omitted in the state space model.

We propose to build a regulator suitable for this problem and then to apply it on the circle following problem. Indeed the glider is supposed to navigate around circles whose radii will be in the range of ten kilometers to a hundred kilometers.

Fig. 5 denotes two consecutive glider states in the case of the circle following problem. By making a first order approximation the following state space model is obtained:

$$
\begin{aligned}
& \dot{d}=v_{c} \sin \beta+v_{g} \sin \delta \\
& \dot{\delta}=\frac{v_{c} \cos \beta+v_{g} \cos \delta}{d}+\omega \\
& \dot{\psi}=\omega .
\end{aligned}
$$

Let $O$ be the center of the circle to be followed (i.e the beacon) and $G$ the glider position. Furthermore let $T$ be the tangent of the circle where the line $O G$ intersects it. We then denote by $\beta$ the angle between this tangent $T$ and the current. As this current is unknown it will be considered as a time varying disturbance. Note that when $d$ tends to infinity

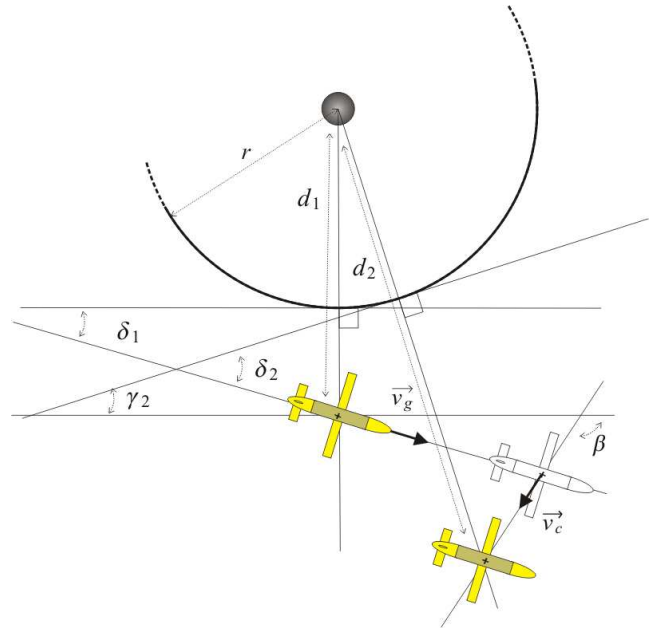

Fig. 5. The new parameters for the system glider - circle

the state space equations become the same as in the case of the line following problem if one considers the line to be followed as the tangent $T$ to the circle.

\section{REgulation}

In this part, the regulator is presented. It is designed to navigate on a circular trajectory of radius $r$ around a float and to be robust to the initial conditions. The guidance stategy chosen to achieve this goal is shown in Fig. 6. An area around the circle is defined by a distance interval $[r-\Delta r, r+\Delta r]$ where a PID (Proportional, Integral and Derivative) regulator is used to follow the desired trajectory. In this area, it is shown that the regulator is very efficient. Outside this interval, the strategy is to reach the reference circle using either homing $(d>r+\Delta r)$ or escape $(d<r-\Delta r)$ algorithms. In this article, even if the homing and escape issues are important, we focus on the case of circle following (i.e. when the glider in the area defined by $[r-\Delta r, r+\Delta r]$ ).

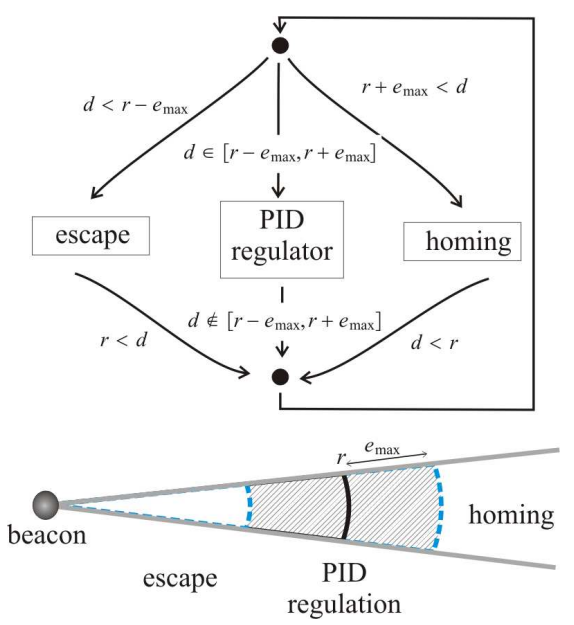

Fig. 6. Regulation diagram

This section presents the controllers used to navigate on a circular trajectory. Due to discrete measurements, the discrete 
form of the controller is used. It is possible to perform the circular guidance by making a control loop on either the heading $\psi$ (based on a magnetic compass) or on the yaw rate $\omega=\dot{\psi}$ (based on a gyroscope).

Consider the error to be minimized:

$$
e_{k}=\frac{d_{k}-r}{\Delta r}
$$

where $\Delta r$ defines the zone where the glider is regulated as seen on Fig. 6.

A PID controller is proposed for the yaw rate $\omega$ in the classical form with $K_{1}$, the integral gain, $K_{2}$ the proportional gain and $K_{3}$ the derivative gain. Note that only $e$ is observed and the derivative term is to be estimated.

The glider makes range measurements every $\Delta T$ time (here $\Delta T$ is 20 minutes). Let us consider the non recursive and recursive form of the discrete controller for the yaw rate:

$$
\begin{aligned}
& I_{k+1}=I_{k}+e_{k+1} \\
& \omega_{k+1}=K_{1} I_{k+1}+K_{2} e_{k+1}+K_{3} \dot{e}_{k+1} \\
& \omega_{k+1}=\omega_{k}+K_{1} e_{k+1}+K_{2}\left(e_{k+1}-e_{k}\right)+K_{3}\left(\dot{e}_{k+1}-\dot{e}_{k}\right)
\end{aligned}
$$

where $\dot{e}_{k}$ is the estimate of the derivative at time step $k$ and $I_{k}$ is the integral term of the error $e_{k}$. As an example, when the data is not noisy one can take the first order approximation:

$$
\dot{e}_{k}=\frac{\left(e_{k+1}-e_{k}\right)}{\Delta T}
$$

By considering the following approximation

$$
\omega_{k+1}=\frac{\psi_{k+1}-\psi_{k}}{\Delta T}
$$

The recursive form for the yaw controller can be derived from the formula for the yaw rate controller:

$$
\psi_{k+1}=\psi_{k}+\Delta T\left(K_{1} I_{k+1}+K_{2} e_{k+1}+K_{3} \dot{e}_{k+1}\right)
$$

Note that the non recursive formula can be easily derived from the recursive one.

Close to the magnetic pole, manetic compasses do not work properly. It would be logical to use a gyroscope to perform this regulation but due to very slow dynamics of the system (sample time is more than 20 minutes) this is not possible without a very precise gyroscope. Then another strategy has to be defined anf the proposed solution is to use the recursive control formulation for the heading:

- make heading corrections (which takes little time: less than a minute) using a gyroscope;

- switch on a open loop mode assuming the glider will move in a straight line. This is true in the fluid frame since the glider is designed in this way (if $\phi=0$ ).

As a consequence, in both gyroscope or compass based guidance, the yaw controller recursive formula (8) is used.

Note that the integral term is not necessary to have a descent trajectory (see simulation results) unless one wants a perfect circle. Therefore the integral term can be discarded. Besides it is an additional constant to fix and there is a need for anti wind-up strategies which adds some additional constraints. In this paper, the keep it simple first approach leads to the choice $K_{1}=0$.

\section{NOISE AND OUTLIERS REJECTION}

The measurements are subject to Gaussian white noise with a standard deviation of $\sigma$. The problem of the noise is that it makes it difficult to estimate the derivatives necessary for guidance. Furthermore, the measurements are corrupted with non-gaussian outliers often due to abnormal propagation of the acoustic signal such as multiple paths trajectories. The proposed algorithm takes into account both noises and outliers. The main idea and contribution is to use robust linear regression on the data. The proposed algorithm uses set membership methods which consider sets of possible values instead of probability distributions in a stochastic approach as mentionned in [14], [15] and [16].

\section{A. Robust regression}

Consider $n$ measurements of an unknown variable $y$ at different times $t_{k}$ denoted $\tilde{y}\left(t_{k}\right), k \in\{1, . ., n\}$. The measurements are subject to noise which is supposed to be contained in an interval $[-\varepsilon, \varepsilon]$. We consider that $\tilde{y}\left(t_{k}\right)$ is not an outliers if

$$
\exists w \in[-\varepsilon, \varepsilon], \tilde{y}\left(t_{k}\right)=y\left(t_{k}\right)+w
$$

The possible values of $y\left(t_{k}\right)$ belong to an interval denoted $\left[y_{k}\right]$ defined as follows

$$
y\left(t_{k}\right) \in\left[\tilde{y}\left(t_{k}\right)-\varepsilon, \tilde{y}\left(t_{k}\right)+\varepsilon\right]=\left[y_{k}\right]
$$

The idea behind set membership linear regression is to find the set of all possible lines which pass through all possible values of $y\left(t_{k}\right), k \in\{1, \ldots, n\}$. If there are no outliers, this consists on finding the set of parameters $(a, b) \in \mathbb{R}^{2}$ which satisfy the following equations

$$
\begin{aligned}
& C_{k}: a *\left(t_{k}-t_{n}\right)+b=y_{k}, \\
& y_{k} \in\left[y_{k}\right], \\
& (a, b) \in \mathbb{R}^{2}, k \in\{1, . ., n\} .
\end{aligned}
$$

This formulation of the problem corresponds to a continuous CSP (Constraint Satisfaction Problem) described in [17]. A CSP is defined by:

- a set of equations (also called constraints) here denoted $C_{1}, . ., C_{n}$,

- a vector of all the variables involved in the problem $\left(a, b, y_{1}, t_{1} \ldots y_{n}, t_{n}\right)$,

- the domains of those different variables.

Solving a CSP consists on finding the parameters $(a, b) \in \mathbb{R}^{2}$ satisfying all the constraints. In case of outliers, this CSP does not admit any solution. Therefore, we search for the parameters $(a, b) \in \mathbb{R}^{2}$ satisfying most of the constraints.

Fig. 7 shows the envelope (in gray) of all the lines consistent with a maximum of measurements (here 3 ) in a simple example involving four measurements with one outlier. The outlier is detected and rejected. The dashed line is the truth.

The CSP solver returns a set of possible values of the parameters $(a, b) \in \mathbb{R}^{2}$. It is usual to consider the center of gravity of that set to perform an position estimation. Note that the CSP solvers are not limited to linear equations. It is 


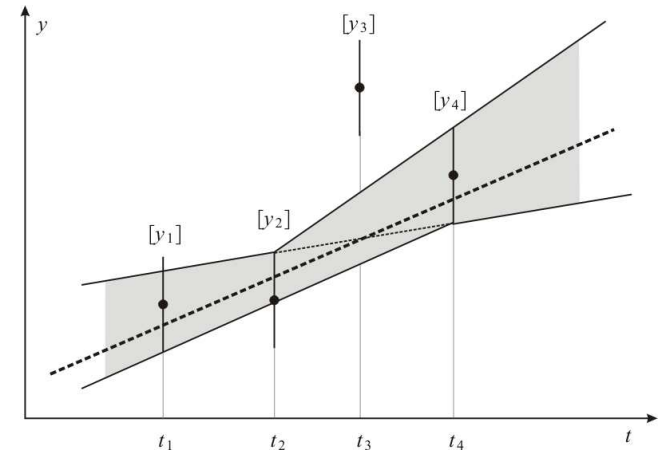

Fig. 7. Example of robust linear regression

possible to use all kind of nonlinear equations such as higher order polynomials or trigonometric functions. We used a linear model for its simplicity.

\section{B. Correcting range measurements}

Consider the last (non missing) $n$ measurements measured with an error in $[-\varepsilon, \varepsilon]$ at the time steps $t_{1}, . ., t_{n}$. If the trajectory was a line, one could apply the robust regression directly on those measurements (which also evolve linearly) to compute the coefficients of the best fit line $a$ and $b$ as explained in the previous subsection. The coefficient $b$ corresponds to the current corrected range measurement. The coefficient $a$ corresponds to an estimate of the derivative of $d$ i.e. the derivative of the error used in the PID controller.

Due to the glider regulation, the trajectory is not a line but a sequence of lines. In order to apply the robust regression, this sequence is transformed into a straight line or a set of parallel lines named a corridor as shown in Fig. 8. The width of the corridor is denoted $\varepsilon_{d}$ and is to be calculated. This value $\varepsilon_{d}$ is then added to the range measurement error which becomes $\left[-\left(\varepsilon+\varepsilon_{d}\right), \varepsilon+\varepsilon_{d}\right]$ and the robust linear regression algorithm can be applied.

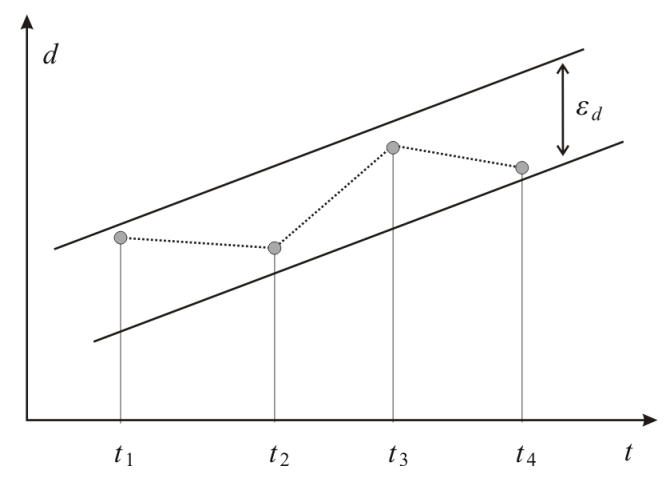

Fig. 8. Fitting the local trajectory in a corridor

The real range values are unknown but their variation is given by the state space equations in (2). In order to determine $\varepsilon_{d}$ we use the following state equations focusing on evolving terms of the glider state equations. We assume $\beta$ is constant due to slow dynamics of the problem:

$$
\begin{aligned}
& \dot{x}=v_{g} \sin y \\
& \dot{y}=u
\end{aligned}
$$

Since the input $u$ is perfectly known, for each initial condition $(x, y)$, it is possible to compute a linear corridor of width $\varepsilon_{d}(x, y)$ enclosing the points $\left.\left(x_{k}, t_{k}\right), k \in\{1, . ., n\}\right)$. These last points are computed by simulation of the state space model (12) and $\varepsilon_{d}(x, y)$ is calculated as follows:

- compute the linear regression,

- the greatest and the least distances between the points and the line determine the width of the corridor $\varepsilon_{d}(x, y)$.

The initial condition on $x$ doesn't affect the computations of $\varepsilon_{d}(x, y)$.

Let us consider the following definition of $\varepsilon_{d}$ :

$$
\varepsilon_{d}=\max \left(\varepsilon_{d}(0, y), y \in\left[-\frac{\pi}{2}, \frac{\pi}{2}\right]\right) .
$$

Note that the max value is obtained for small $y(\delta=0$ maximizes the variations of the acceleration of $d$ ).

\section{Simulation RESUlTS}

For the initialization, the glider needs a series of $N$ measurements to get an estimation of distance and its derivative. In this phase, one solution is to make the glider go straight line for $N$ measurements (here 2 hours) and then the guidance is launched with respect to the circle of the radius corresponding to the last distance estimation. Fig. 9 shows simulation results for the following parameters:

$$
\begin{aligned}
& v_{g}=0.3 \mathrm{~m} / \mathrm{s}, v_{c}=0.15 \mathrm{~m} / \mathrm{s}, \alpha=-\frac{\pi}{2} \\
& r=10 \mathrm{~km}, \Delta r=3 \mathrm{~km}, \\
& \varepsilon=150 \mathrm{~m}, \text { maxoutliers }=40 \%, \text { missing }=10 \% \\
& K_{1}=0, K_{2}=0.1, K_{3}=0.7, n=7
\end{aligned}
$$

The gray lines are the measurements, the black dots are the trajectory. In order to increase the area where the glider

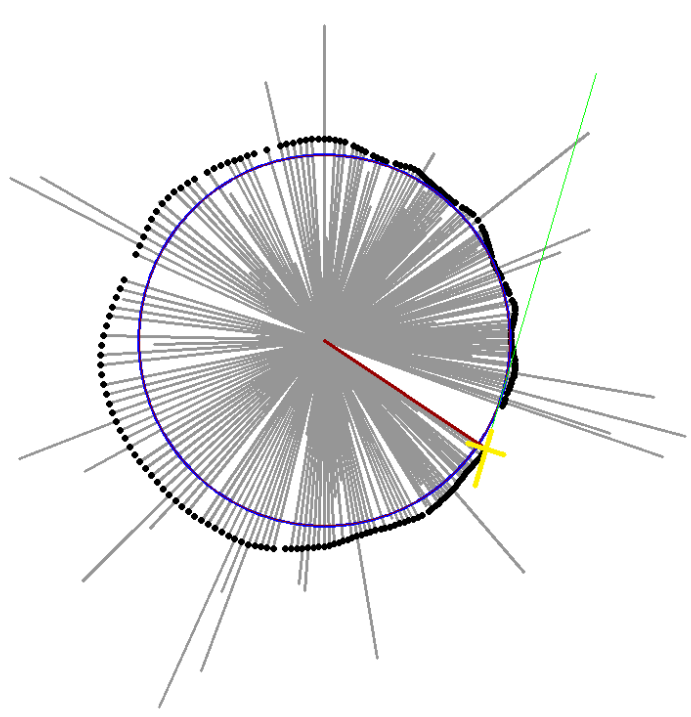

Fig. 9. Result of regulation

makes measurements, the glider can follow a helicoidal trajectory as shown in Fig. 10. The outliers and the missing data can clearly be seen on the image. In the presented simulation, 
the glider performs an helicoidal trajectory starting at 20 $\mathrm{km}$ and ending at $5 \mathrm{~km}$ with a step of $3 \mathrm{~km}$. The mean accuracy of the method is about 900 meters. Although the precision is not very high, the most important is to stay close to the desired trajectory since the localization is done in post processing.

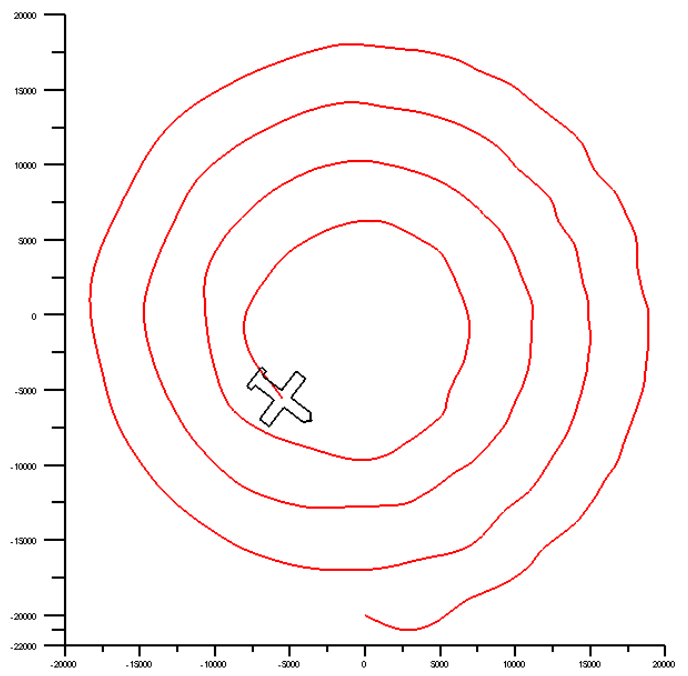

Fig. 10. Helicoidal trajectory to cover a wide area (distances in meters)

\section{VALIDATION}

Validation is performed with simulations; the Fig. 11 shows the trajectory of the robot for a duration of one year around a circle of $10 \mathrm{~km}$ radius. The robot has not even quit the circular trajectory once.

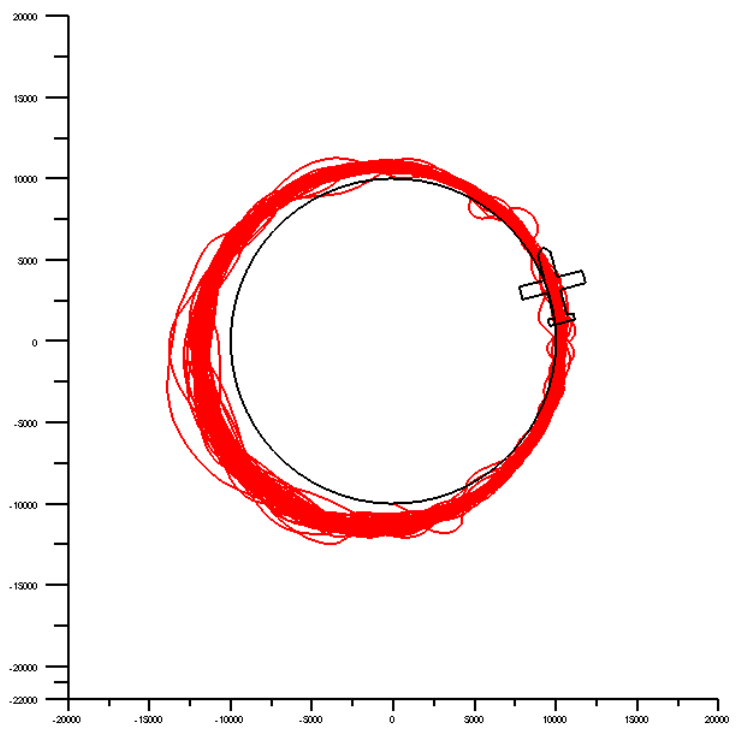

Fig. 11. One year navigation (distances in meters)

\section{CONCLUSION}

As for now, the main point is that this algorithm satisfies the requirements of guidande, robusteness and simplicity. The algorithm can be improved using more advanced techniques such as a better regulator than a PID. The best tuning can be found through simulations and should be good approximations for the real glider. The algorithm can be validated using methods described in II. Finally, many assumptions have been made concerning the nature of the data (noise evolution with distance, outliers rate, missing data rate...) which are to be confirmed through experiments. It is important to note that the presented approach is to control the glider so that it maintains a certain distance from the master beacon (equipped with a transponder), and to defer localization to postprocessing. However, it is important to include in a future work the alternate methods for comparision in terms of computational cost and efficiency.

\section{REFERENCES}

[1] S. Sandven, "ACOBAR - acoustic technology for observing the interior of the arctic ocean," http://acobar.nersc.no, 2009.

[2] T. Rossby, D. Dorson, and J. Fontaine, "The RAFOS system," Journal of Atmospheric and Oceanic Technology, vol. 3, pp. 672-680, 1986.

[3] D. Manolakis, "Efficient solution and performance analysis of 3-d position estimation by trilateration," IEEE Transactions on Aerospace and Electronic Systems, vol. 32, pp. 1239-1248, 1996.

[4] O. Hegrenas, K. Gade, O. Hagen, and P. Hagen, "Underwater transponder positioning and navigation of autonomous underwater vehicles," in Proceedings of MTS/IEEE OCEANS 2009, Biloxi, USA, 2009.

[5] P. Baccou and B. Jouvencel, "Homing and navigation using one transponder for AUV, postprocessing comparisons results with long base-line navigation," in Proceedings of IEEE International Conference on Robotics and Automation ICRAO2, vol. 4, 2002, pp. 4004 4009.

[6] M. Larsen, "Synthetic long baseline navigation of underwater vehicles," in Proceedings of OCEANS 2000 MTS/IEEE Conference and Exhibition, vol. 3, 2000, pp. 2043-2050.

[7] C. E. G. LaPointe, "Virtual long baseline (VLBL) autonomous underwater vehicle navigation using a single transponder," Master's thesis, Massachusetts Institute of Technology. Dept. of Mechanical Engineering, 2006.

[8] B. Ferreira, A. Matos, and N. Cruz, "Single beacon navigation: Localization and control of the MARES AUV," in Proceedings of MTS/IEEE conference OCEANS 2010, 2010, pp. 1-9.

[9] J. Aubin, Viability Theory. Boston-Birkhauser, 1991.

[10] D. Webb, P. Simonetti, and C. Jones, "SLOCUM: an underwater glider propelled by environmental energy," IEEE Journal of Oceanic Engineering, vol. 26, no. 4, pp. 447-452, 2001.

[11] C. Eriksen, T. Osse, R. Light, T. Wen, T. Lehman, P. Sabin, J. Ballard, and A. Chiodi, "Seaglider: a long-range autonomous underwater vehicle for oceanographic research," IEEE Journal of Oceanic Engineering, vol. 26, no. 4, pp. $424-436$, oct 2001.

[12] R. Moitie and N. Seube, "Guidance and control of an autonomous underwater glider," in Proceedings of IEEE 12th Int. Symposium on Unmanned Untethered Submersible, Durham, NH, USA, 2001.

[13] F. Floc'h, "Prédiction de trajectoires d'objets immergés par couplage entre modèles d'écoulement et équations d'Euler-Newton," Ph.D. dissertation, ENSTA Bretagne and UBO, 2011.

[14] J. Sliwka, "Using set membership methods for robust underwater robot localization,” Ph.D. dissertation, ENSTA Bretagne and UBO, 2011.

[15] J. Sliwka, F. Le Bars, O. Reynet, and L. Jaulin, "Using interval methods in the context of robust localization of underwater robots," in Proceedings of IEEE NAFIPS11 conference, El Paso, Texas, 2011.

[16] L. Jaulin, "Robust set membership state estimation ; application to underwater robotics," Automatica, vol. 45, no. 1, pp. 202-206, 2009.

[17] K. Apt, Principles of Constraint Programming. Cambridge University Press, 2003 